\title{
3-DOF Trilateral Teleoperation Using A Pair of 1-DOF and 2-DOF Haptic Devices: Stability Analysis
}

\author{
Jian Li ${ }^{*, * *}$ Mahdi Tavakoli ${ }^{* *}$ Qi Huang ${ }^{* * *}$ \\ ${ }^{*}$ School of Energy Science and Engineering, University of Electronic \\ Science and Technology of China, Chengdu, Sichuan, China 611731 \\ (e-mail: jian1@ualberta.ca). \\ ** Department of Electrical and Computer Engineering, University of \\ Alberta, Edmonton, AB T6G 2V4, Canada (e-mail: \\ mahdi.tavakoli@ualberta.ca) \\ *** School of Energy Science and Engineering, University of Electronic \\ Science and Technology of China, Chengdu, Sichuan, China 611731 \\ (e-mail: huangqi@uestc.edu.cn)
}

\begin{abstract}
Humans are usually better than autonomous robots in operating in complex environments. In bilateral teleoperation, to take full advantage of the human's intelligence, experience, and sensory inputs for performing a dexterous task, a possibility is to use the two hands of the user to manipulate two master haptic devices in order to control a slave robot with multiple degrees-of-freedom (DOF); the total DOFs of the two masters are equal to the DOFs of the slave. In this paper, two 1-DOF and 2-DOF haptic robots are considered as the two masters while a 3-DOF robot acts as the slave in a trilateral teleoperation system. It is discussed how such a system can result in better task performance by splitting the various DOFs of a dexterous task between two hands or two users, e.g., during peg-in-the-hole insertion. The stability analysis of such a system is not trivial due to dynamic coupling across the different DOFs of the robots, the human operators, and the physical/virtual environments. Also, the unknown dynamics of the users and the environments exacerbate the problem. We present a novel, straightforward and convenient method for stability analysis of this teleoperation system. Simulation results demonstrate the validity of the approach.
\end{abstract}

Keywords: Absolute stability, multi-master/single-slave teleoperation.

\section{INTRODUCTION}

Robotic manipulators with multiple degrees of freedom (DOF) have recently found many applications such as in robotic-assisted surgery and therapy, space exploration, and navigation systems (Tanaka et al. (2009); Ueki et al. (2012)). When a multi-DOF manipulator is acting as the slave robot in a master-slave haptic teleoperation system, the human controls it and receives haptic feedback via a master robot typically with the same DOFs. For successful performance of a multi-DOF task in a physical environment by the human operator of a teleoperation system, two assumptions are made. First, it is assumed that the human is always in the loop, i.e., every move made to the master by the human has been informed by continuous visual and haptic updates received from the slave. Second and more importantly, it is assumed that the teleoperator (the electromechanical system comprising the master, the slave, the controllers for the master and the slave, and the communication channel between the master and the slave) is transparent. Under these two assumptions, the human should feel as if he/she is doing the multi-DOF task via direct touch with the environment while actually doing it via the teleoperation system. Therefore, it is expected that the natural capabilities of the human in terms of execution of multi-DOF tasks is transferred to teleoperation. Even more, it is expected that due to super-human capabilities of machines such as tremor filtering, high-accuracy positioning, and motion or force scaling, the task performance in teleoperation is better than that in direct touch.

In practice, while the teleoperator amplifies certain skills of the human, it may attenuate some other skills. Moreover, despite the vast amount of research aimed at teleoperation transparency enhancement in recent years, there are still many electromechanical transparency-limiting imperfections in teleoperators including delays, uncertainties, nonlinearities, sampling, quantization (Kim et al. (2007)), and switching. (Walker et al. (2009)). As a result, the performance of multi-DOF tasks via teleoperation is not as simple as it should be.

Haptic virtual fixtures as software-generated forces have been used to guide the human through a task with a specified path. A virtual fixture effectively creates motion constraints in a subset of the haptic device's DOFs, allowing the human to focus on the remaining DOFs and thus resulting in improved task performance. An informative examples is given in Hager (2010). For retinal 
vein cannulation, a needle of about 20-50 $\mu \mathrm{m}$ in diameter must be inserted into the lumen of a retinal vein, which is about $100 \mu \mathrm{m}$ in diameter. Given the very small scale at which the task is to be executed, there is a need to drastically enhance the precision of human motion. A virtual fixture can do so by constraining the needle motion in the lateral directions to stabilize the human hand while allowing the needle motion in the axial direction.

As explained before, virtual fixtures deliberately but perhaps temporarily eliminate a subset of the haptic device's DOFs in order to improve the performance of a multiDOF task. An alternative strategy considered in this paper is to allow the human to use his/her two hands to manipulate two haptic devices, each of which provides a subset of the DOFs required in the task. This can offer advantages to single-handed, virtual-fixture-based assistance. First, with multi-DOF robots such as the Jaco arm (Kinova Robotics, Montreal, Canada) for performing dextrous manipulation tasks by the disabled, two haptic devices help make most of the limited but possibly complementary motions of the patient's two hands. Second, using two haptic devices allows to separate the required motions in a multi-DOF task into gross vs. fine, positioncontrolled vs. force-controlled, translational vs. rotational, fast vs. slow, etc. This separation has the potential to improve dexterous task performance. Third, building a highly-dexterous master for every new application can be very costly whereas combining the capabilities of two lessdexterous, off-the-shelf masters can be a more affordable solution. These provide the motivation for considering twohanded cooperative teleoperation systems that involve two haptic devices with complementary degrees of freedom (Zhai and Milgram (1998)).

Closed-loop system stability is critical for safe and effective teleoperation. However, investigation of teleoperation system stability using common closed-loop stability analysis tools in the control systems literature is not possible because the models of the human and the environment are usually unknown, uncertain, and/or time-varying. Research has shown that it is still possible to draw stability conditions for a haptic teleoperation system under unknown human and environment as long as they are passive (Li et al. (2013a,b)). While this is referred to as absolute or unconditional stability in the literature, we simply call it stability in this paper.

In this paper, we present a new approach for stability analysis of two-handed cooperative trilateral teleoperation systems with two haptic devices with complementary DOFs. For simplicity, we consider a $1-\mathrm{DOF}$ robot and a 2 -DOF robot as the two masters and a 3 -DOF robot as the slave in a trilateral teleoperation system; we will call this (1+2)-DOF dual-master/3-DOF single-slave teleoperation. We then use the proposed stability criterion to design stabilizing teleoperation controllers for the system.

The rest of the paper is organized as follows: The next section gives mathematical definitions and lemmas for analysis of stability. Section 3 propose a $(1+2)$-DOF dualmaster/3-DOF single-slave teleoperation system and introduces the peg-in-the-hole task. Next, in Section 4, the proposed stability analysis method from network theory is derived. Then, as a case study to show how the feasibility

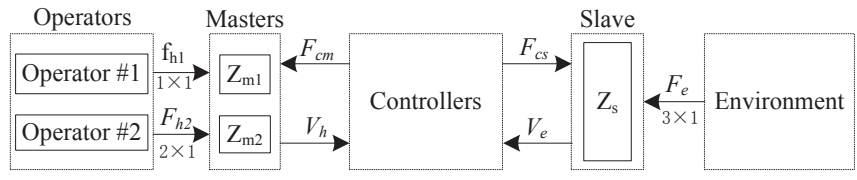

Fig. 1. A multiple masters $/ m$-DOF slave teleoperation system

of the approach can be utilized, in Section 5, a 1-DOF +2 -DOF dual-master/3-DOF single slave teleoperation system with position-position control is considered, the stability conditions in terms of system parameters including controller gains are found, and simulations to verify the validity of the calculated stability conditions are presented. Section 6 contains concluding remarks.

\section{MATHEMATICAL PRELIMINARIES}

Notation 1. a is a scalar, $A$ is a vector, $\mathbf{A}$ is a matrix, and $\mathcal{A}$ is a block matrix (i.e., with matrix elements).

Definition 2. A two-port network is passive if the total energy delivered to the network at its ports is nonnegative.

Definition 3. (Khalil (2002)) A $n \times n$ proper rational transfer matrix $\mathbf{G}(\mathbf{s})$ is positive real if

i) Poles of all elements of $\mathbf{G}(\mathbf{s})$ are in $\operatorname{Re}[s] \leq 0$,

ii) Any pure imaginary pole $j \omega$ of any element of $\mathbf{G}(\mathbf{s})$ is a simple pole and the residue matrix $\lim _{s \rightarrow j \omega}(s-j \omega) \mathbf{G}(\mathbf{s})$ is positive semidefinite Hermitian,

iii) For all real $\omega$ for which $j \omega$ is not a pole of any element of $\mathbf{G}(\mathbf{s})$, the matrix $\mathbf{G}(j \omega)+\mathbf{G}^{T}(-j \omega)$ is positive semidefinite.

Property 4. A Hermitian matrix, i.e., a square matrix equal to its conjugate transpose, is positive definite if its leading principal minors are all positive.

Lemma 5. (Khalil (2002)) A linear time-invariant minimal realization model with transfer matrix $\mathbf{G}(\mathbf{s})$ is passive if $\mathbf{G}(\mathbf{s})$ is positive real.

Definition 6. A two-port network is absolutely stable if the coupled system remains bounded-input boundedoutput stable under all possible passive terminations. Otherwise, it is potentially unstable.

Lemma 7. (Youla (1959)) Let $\mathbf{Z}=\mathbf{Z}^{\mathrm{T}}$ be the impedance matrix of a reciprocal $n$-port network. Then, the network is passive if and only if it is absolutely stable.

Lemma 8. (Youla (1960)) Let $\mathbf{Z}_{\mathbf{1}}$ and $\mathbf{Z}_{\mathbf{2}}$ be the impedance matrices of two $n$-port networks. Then, if $\mathbf{Z}_{\mathbf{1}}$ and $\mathbf{Z}_{\mathbf{2}}$ possess identical principal minors of all orders, then $\mathbf{Z}_{\mathbf{1}}$ is absolutely stable if and only if $\mathbf{Z}_{\mathbf{2}}$ is absolutely stable.

An $n$-port network is stable if the port currents $I_{1}, I_{2}, \cdots, I_{n}$ are zero under all passive terminations $z_{1}, z_{2}, \cdots, z_{n}$ for ports (Youla (1959)). In other words, an $n$-port network with an impedance matrix $Z_{n \times n}$ is stable if and only if the equation $\left(Z+Z_{0}\right) \mathbf{I}=0$, where $I=\left[I_{1}, I_{2}, \cdots, I_{n}\right]^{T}$ and $Z_{0}=\operatorname{diag}\left[z_{1}, z_{2}, \cdots, z_{n}\right]$ has only the trivial solution $\mathbf{I}=0$ for every passive choice of $Z_{0}$; this happens if and only if $\operatorname{det}\left(Z+Z_{0}\right) \neq 0$. On the other hand, according to Youla (1960), if two $n \times n$ matrices $Z_{1}$ and $Z_{2}$ have identical principal minors of all orders, then

$$
\operatorname{det}\left(Z_{1}+Z_{0}\right)=\operatorname{det}\left(Z_{2}+Z_{0}\right)
$$


for any $Z_{0}=\operatorname{diag}\left[z_{1}, z_{2}, \cdots, z_{n}\right]$. This implies that the stability of two $n$-port networks with impedance matrices $Z_{1}$ and $Z_{2}$ will happen at the same time (Lemma 8).

\section{A TELEOPERATION SYSTEM WITH TWO HAPTIC DEVICES WITH COMPLEMENTARY MOTIONS}

The proposed (1+2)-DOF dual-master/3-DOF singleslave teleoperation system is shown in Figure 1. To analyze the stability of such a teleoperation system, first the possibly nonlinear dynamics of the 1-DOF master (master 1), the 2-DOF master (master 2), and the 3-DOF slave need to be modeled around their operating points by linear-timeinvariant (LTI) impedances $z_{m 1}(1 \times 1), \mathbf{Z}_{m 2}(2 \times 2)$, and $\mathbf{Z}_{s}$ $(3 \times 3)$, respectively. These impedances either relate joint torques to joint angular velocities or end-effector forces to end-effector Cartesian velocities; without loss of generality, we will assume the latter is the case in the rest of the paper. Then, the teleoperation system is modeled as

$$
\begin{aligned}
\mathcal{Z}_{m} V_{h} & =F_{h}+F_{c m} \\
\mathbf{Z}_{s} V_{e} & =F_{e}+F_{c s}
\end{aligned}
$$

where $\mathcal{Z}_{m}=\operatorname{diag}\left[z_{m 1}, \mathbf{Z}_{m 2}\right]$. Also, $F_{h}=\left[f_{h 1}{ }^{\prime} f_{h 2} f_{h 3}\right]^{T}$ denotes the interaction force vector between the two humans (or the two hands of the same human) and the two masters and $F_{e}=\left[\begin{array}{lll}f_{e 1} & f_{e 2} & f_{e 3}\end{array}\right]^{T}$ denotes the interaction force vector between the slave and the environment. Furthermore, $V_{h}=\left[\begin{array}{lll}v_{h 1}, v_{h 2} & v_{h 3}\end{array}\right]^{T}$ and $V_{e}=\left[\begin{array}{lll}v_{e 1} & v_{e 2} & v_{e 3}\end{array}\right]^{T}$ are the human and the environment velocity vectors while $F_{c m}=\left[\begin{array}{lll}f_{c m 1}{ }^{\prime} & f_{c m 2} & f_{c m 3}\end{array}\right]^{T}$ and $F_{c s}=\left[\begin{array}{lll}f_{c s 1} & f_{c s 2} & f_{c s} 3\end{array}\right]^{T}$ denote the control signals sent to the two master and the slave, respectively.

For such a $(1+2)$-DOF dual-master/3-DOF single-slave teleoperation system, planar peg-in-the-hole insertion is a an interesting manipulation task (Yamashita et al. (1991)). As shown in Figure 2, this is a 3-DOF task that involves two translations $(y$ and $z$ ) and one rotation $(\phi)$. We will consider two distinct cases: (i) the 1-DOF master 1 is manipulated by a hand of the human to control $\phi$ while the 2-DOF master 2 is manipulated by the other hand of the human to control $y$ and $z$; (ii) A 3-DOF master is manipulated by a single hand of the human to control the $y, z$ and $\phi$. In both cases, a 3-DOF slave robot holds the peg and performs the $y, z$ and $\phi$ maneuvers based on the position commands from the master(s).

In case (i), as shown in Figure 2, the procedure is divided into three steps. In the first step, the peg is moved at an angle toward the hole; this step is completed when the peg makes contact with the edge of the hole. In the second step, an insertion force is applied in the $z$ direction and a force is applied in the $y$ direction to maintain the contact between the peg and the hole's edge. At the same time, the peg is turned along the $\phi$ direction to become co-axial to the hole axis. This step is completed once the peg is aligned with and slightly inside the hole. In the third step, an insertion force in the $z$ direction is applied while the lateral force in the $y$ direction and the moment in the $\phi$ direction are kept to zero. Clearly, master 2 can control the first and the third steps while master 1 can control the second step, providing a tangible separation between the translational and rotational DOFs in order to simplify the performance of this dexterous task. On the other hand, in

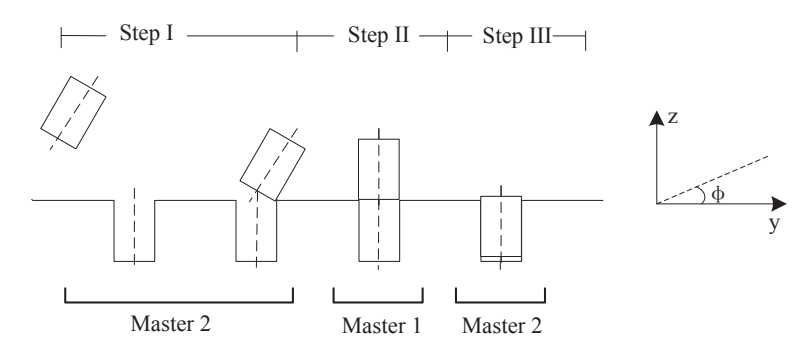

Fig. 2. The insertion procedure.

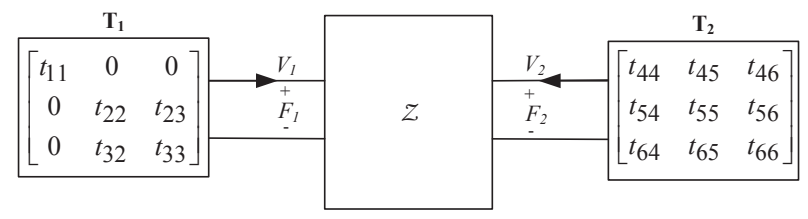

Fig. 3. A two-port network with each port connected to a 3-DOF termination.

case (ii), the procedure can take longer because the peg can more easily be jammed inside the hole. In fact, in the third step, the human may cause $\phi$ motions when he/she tries to apply $z$ motions. Obviously, two-handed teleoperation is more accurate for this task.

\section{MAIN RESULT: AN STABILITY CRITERION FOR 1-DOF + 2-DOF DUAL-MASTER/3-DOF SINGLE-SLAVE TELEOPERATION SYSTEMS}

A (1+2)-DOF dual-master/3-DOF single-slave teleoperation system can be modeled as a two-port network where each port (terminal) connects to a 3 -DOF termination as shown in Figure 3. The network impedance model will be

$$
F=\mathcal{Z} V
$$

where

$$
F=\left[\begin{array}{ll}
F_{1} & F_{2}
\end{array}\right]^{T}, \quad V=\left[\begin{array}{ll}
V_{1} & V_{2}
\end{array}\right]^{T}
$$

and $F_{i}$ and $V_{i}, i=1,2$, represent the $3 \times 1$ vectors of force and velocity at the $i^{\text {th }}$ port of the network, respectively. The impedance matrix of the network is

$$
\mathcal{Z}=\left[\begin{array}{ll}
\mathbf{Z}_{11} & \mathbf{Z}_{12} \\
\mathbf{Z}_{21} & \mathbf{Z}_{22}
\end{array}\right]=\left[\begin{array}{ccc}
z_{1,1} & \cdots & z_{1,6} \\
\vdots & \ddots & \vdots \\
z_{6,1} & \cdots & z_{6,6}
\end{array}\right]
$$

where $\mathbf{Z}_{i j}, i, j=1,2$, are the following $3 \times 3$ matrices: $(6)$.

$$
\mathbf{Z}_{i j}=\left[\begin{array}{ccc}
z_{3 i-2,3 j-2} & z_{3 i-2,3 j-1} & z_{3 i-2,3 j} \\
z_{3 i-1,3 j-2} & z_{3 i-1,3 j-1} & z_{3 i-1,3 j} \\
z_{3 i, 3 j-2} & z_{3 i, 3 j-1} & z_{3 i, 3 j}
\end{array}\right]
$$

On the other hand, the pair of 3-dimensional terminations is represented by

$$
\mathcal{T}=\operatorname{diag}\left[\mathbf{T}_{1}, \mathbf{T}_{2}\right]
$$

where $\mathbf{T}_{i}, i=1,2$, represents the $3 \times 3$ impedance matrix of the $i^{\text {th }} m$-dimensional termination.

Let

$$
\mathcal{Z}^{\prime}=\left[\begin{array}{cccc}
z_{1,1} & \gamma_{1} \sqrt{z_{1,2} z_{2,1}} & \cdots & \gamma_{5} \sqrt{z_{1,6} z_{6,1}} \\
\gamma_{1} \sqrt{z_{1,2} z_{2,1}} & z_{2,2} & \cdots & \gamma_{9} \sqrt{z_{2,6} z_{6,2}} \\
\vdots & \vdots & \cdots & \vdots \\
\gamma_{5} \sqrt{z_{1,6} z_{6,1}} & \gamma_{9} \sqrt{z_{2,6} z_{6,2}} & \cdots & z_{6,6}
\end{array}\right]
$$


where $\gamma_{i}= \pm 1$ and $i=1,2, \cdots, 15$. The following theorem is the main result in this paper.

Theorem 9. A (1+2)-DOF dual-master/3-DOF single-slave teleoperation system with impedance matrix $\mathcal{Z}$ in (5) satisfying the symmetrization conditions

A) $z_{i, j} z_{j, k} z_{k, i}=z_{j, i} z_{k, j} z_{i, k}$, where $i, j, k=1,2, \cdots, 6$, $i \neq j \neq k$, and $i \neq k$.

B) $\mathbf{Z}_{\ell \ell}$ is symmetric, where $\ell=1,2$.

is absolutely stable if and only if

C) The elements of $\mathcal{Z}$ in (6) have no poles in the righthalf plane (RHP)

D) Any poles of the elements of $\mathcal{Z}$ in (6) on the imaginary axis are simple, and the leading principal minors of the residues matrix of $\mathcal{Z}$ at these poles are greater than zero.

E) For all real values of frequencies $\omega$, the leading principal minors of the real part of $\mathcal{Z}^{\prime}$ in (8) are greater than or equal to zero, i.e.,

$$
\begin{aligned}
& \operatorname{Re}\left(z_{i, i}\right) \geq 0, \quad i=1,2, \cdots n \times m \\
& \operatorname{Re}\left(z_{1,1}\right) \operatorname{Re}\left(z_{2,2}\right)-\frac{\left|z_{1,2} z_{2,1}\right|+\operatorname{Re}\left(z_{1,2} z_{2,1}\right)}{2} \geq 0
\end{aligned}
$$

$$
\operatorname{det}\left(\operatorname{Re}\left(\mathcal{Z}^{\prime}\right)\right) \geq 0
$$

Proof. Consider a linear time-invariant system with impulse response $h(t)$. The system's transfer function is the Laplace transform of $h(t)$ defined as

$$
H(s)=\int_{0}^{\infty} h(t) e^{-s t} d t
$$

where $s=\sigma+j \omega . H(s)$ is stable if every bounded input produces a bounded output and this happens if the poles of $H(s)$ have negative real parts. This stability definition is equivalent to the absolute convergence (defined below) of $H(s)$ in the region $\operatorname{Re}(s) \geq 0$. If $h$ is locally integrable, then $H(s)$ is said to converge if the limit $H(s)=\lim _{r \rightarrow \infty} \int_{0}^{r} h(t) e^{-s t} d t$ exists. Also, $H(s)$ is said to converge absolutely if the integral $\int_{0}^{\infty}\left|h(t) e^{-s t}\right| d t$ exists. The set of values of $s$ for which $H(s)$ converges is known as the region of convergence (ROC) and is of the form $\operatorname{Re}(s) \geq a$, where $a$ is a real constant. Importantly, if $H(s)$ converges at $s=s_{0}$, then it will converge for all $s$ with $\operatorname{Re}(s)>\operatorname{Re}\left(s_{0}\right)$. The above means that for stability analysis it suffices to focus on the convergence of $H(s)$ when $\operatorname{Re}(s)=0$, i.e., on the $j \omega$ axis. This is sometimes referred to as real-frequency stability. Thus, as a linear time-invariant system, the stability of a $(1+2)$-DOF dualmaster/3-DOF single-slave teleoperation system needs to be analyzed only for $s=j \omega$.

According to Lemma 8, if there exists a reciprocal $n$-port network with impedance matrix $\mathcal{Z}^{\prime}$ that has the same stability characteristics as the original nonreciprocal $n$ port network with the impedance matrix $\mathcal{Z}$, then

$$
\operatorname{det}\left(\mathcal{Z}^{\prime}+\mathcal{T}\right)=\operatorname{det}(\mathcal{Z}+\mathcal{T})
$$

for any passive $\mathcal{T}$ in (7). Thus,

$$
\operatorname{det}\left[\begin{array}{cc}
\mathbf{Z}_{11}^{\prime}+\mathbf{T}_{1} & \mathbf{Z}_{12}^{\prime} \\
\mathbf{Z}_{21}^{\prime} & \mathbf{Z}_{22}^{\prime}+\mathbf{T}_{2}
\end{array}\right]=\operatorname{det}\left[\begin{array}{cc}
\mathbf{Z}_{11}+\mathbf{T}_{1} & \mathbf{Z}_{12} \\
\mathbf{Z}_{21} & \mathbf{Z}_{22}+\mathbf{T}_{2}
\end{array}\right]
$$

The above is to hold for any passive $\mathcal{T}$. It is easy to show that calculating the two determinants and equating the coefficients of $\mathbf{T}_{1}, \mathbf{T}_{2}$ gives the matrix $\mathcal{Z}^{\prime}$ in (8) as well as the symmetrization conditions $\mathrm{A}$ and $\mathrm{B}$.

On the other hand, according to Lemma 7, the reciprocal $n$-port network with impedance matrix $\mathcal{Z}^{\prime}$ is absolutely stable if and only if it is passive. In turn, according to Lemma $5, \mathcal{Z}^{\prime}$ is passive if and only if it is positive real, which can be verified through Definition 3.

From the above, we conclude that the original nonreciprocal $n$-port network with impedance matrix $\mathcal{Z}$ is absolutely stable if and only if the equivalent reciprocal $n$-port network's impedance matrix $\mathcal{Z}^{\prime}$ is positive real. In this context, it is straightforward to show that Conditions $\mathrm{C}$ and $\mathrm{D}$ in Theorem 9 are the same as Conditions i) and ii) in Definition 3. Also, according to Condition iii) of Definition 3, the Hermitian matrix

$$
\mathcal{Z}^{\prime}(j \omega)+\mathcal{Z}^{\prime T}(-j \omega)=2 \operatorname{Re}\left(\mathcal{Z}^{\prime}(j \omega)\right)
$$

needs to be positive semidefinite for the $n$-port network with impedance matrix $\mathcal{Z}$ to be absolutely stable. Using Property 4, and simplifying the conditions by

$$
\left(\operatorname{Re}\left(\sqrt{z_{i, j} z_{j, i}}\right)\right)=\sqrt{\frac{\left|z_{i, j} z_{j, i}\right|+\operatorname{Re}\left(z_{i, j} z_{j, i}\right)}{2}}
$$

where $i, j=1,2, \cdots, 6$, we arrive at conditions (9a)-(9c). This concludes the proof.

\section{CASE STUDY: STABILITY OF A 1-DOF + 2-DOF DUAL-MASTER/3-DOF SINGLE-SLAVE TRILATERAL TELEOPERATION SYSTEM}

In this section, the aim is to apply the proposed stability criterion to a $(1+2)$-DOF dual-master/3-DOF single-slave teleoperation system. Then, simulations will be conducted for verifying the theoretical stability conditions.

\subsection{A dual-master/single-slave teleoperation system}

In a $(1+2)$-DOF dual-master/3-DOF single-slave teleoperation system, the equations of motion of the two masters and the slave in contact with the humans and the environment, respectively, were shown in Section 3. In this case study, we assume the three dimensions are $x$ (for the 1-DOF master 1 ) and $y$ and $z$ (for the 2-DOF master 2). Obviously, the previous definitions changes to $F_{h}=\left[\begin{array}{lll}f_{h x}{ }^{\prime}, f_{h y} & f_{h z}\end{array}\right]^{T}, F_{e}=\left[\begin{array}{lll}f_{e x} & f_{e y} & f_{e z}\end{array}\right]^{T}, V_{h}=$ $\left[\begin{array}{lll}v_{h x}{ }^{\prime} v_{h y} & v_{h z}\end{array}\right]^{T}$, and $V_{e}=\left[\begin{array}{lll}v_{e x} & v_{e y} & v_{e z}\end{array}\right]^{T}$. Modeling each robot by a mass, where $\mathbf{Z}_{m}=\mathbf{M}_{m} s$, and $\mathbf{Z}_{s}=\mathbf{M}_{s} s$, we have

$$
\mathbf{M}_{m}=\left[\begin{array}{ccc}
m_{m x x} & 0 & 0 \\
0 & m_{m y y} & m_{m y z} \\
0 & m_{m y z} & m_{m z z}
\end{array}\right] \quad \mathbf{M}_{s}=\left[\begin{array}{ccc}
m_{s x x} & m_{s x y} & m_{s x z} \\
m_{s x y} & m_{s y y} & m_{s y z} \\
m_{s x z} & m_{s y z} & m_{s z z}
\end{array}\right]
$$

Let us consider position-position control laws for teleoperation (Tavakoli et al. (2007)):

$$
\begin{aligned}
F_{c m} & =-\mathbf{C}_{m} V_{h}+\mathbf{C}_{4} V_{e} \\
F_{c s} & =-\mathbf{C}_{s} V_{e}+\mathbf{C}_{1} V_{h}
\end{aligned}
$$


where, due to representing all impedances in the forcevelocity domain rather than the force-position domain, the normally PD position controllers show up as PI velocity controllers:

$$
\begin{aligned}
& \mathbf{C}_{m}=\left[\begin{array}{ccc}
\frac{k_{p m x x}+k_{v m x x} s}{s} & 0 & 0 \\
0 & \frac{k_{p m y y}+k_{v m y y} s}{s} & \frac{k_{p m y z}+k_{v m y z} s}{s} \\
0 & \frac{k_{p m y z}+k_{v m y z} s}{s} & \frac{k_{p m z z}+k_{v m z z} s}{s}
\end{array}\right] \\
& \mathbf{C}_{s}=\left[\begin{array}{llll}
\frac{k_{p s x x}+k_{v s x x} s}{s} & \frac{k_{p s x y}+k_{v s x y} s}{s} & \frac{k_{p s x z}+k_{v s x z} s}{s} \\
\frac{k_{p s x y}+k_{v s x y} s}{s} & \frac{k_{p s y y}+k_{v s y y} s}{s} & \frac{k_{p s y z}+k_{v s y z} s}{s} \\
\frac{k_{p s x z}+k_{v s x z} s}{s} & \frac{k_{p s y z}+k_{v s y z} s}{s} & \frac{k_{p s z z}+k_{v s z z} s}{s}
\end{array}\right] \\
& \mathbf{C}_{4}=\left[\begin{array}{ccc}
\frac{k_{p 4 x x}+k_{v 4 x x} s}{s} & 0 & 0 \\
0 & \frac{k_{p 4 y y}+k_{v 4 y y} s}{s} & \frac{k_{p 4 y z}+k_{v 4 y z} s}{s} \\
0 & \frac{k_{p 4 y z}+k_{v 4 y z} s}{s} & \frac{k_{p 4 z z}+k_{v 4 z z} s}{s}
\end{array}\right] \\
& \mathbf{C}_{1}=\left[\begin{array}{llll}
\frac{k_{p 1 x x}+k_{v 1 x x} s}{s} & \frac{k_{p 1 x y}+k_{v 1 x y} s}{s} & \frac{k_{p 1 x z}+k_{v 1 x z} s}{s} \\
\frac{k_{p 1 x y}+k_{v 1 x y} s}{s} & \frac{k_{p 1 y y}+k_{v 1 y y} s}{s} & \frac{k_{p 1 y z}+k_{v 1 y z} s}{s} \\
\frac{k_{p 1 x z}+k_{v 1 x z} s}{s} & \frac{k_{p 1 y z}+k_{v 1 y z} s}{s} & \frac{k_{p 1 z z}+k_{v 1 z z} s}{s}
\end{array}\right]
\end{aligned}
$$

By substituting (15) in (2), the impedance matrix of the $(1+2)$-DOF dual-master/3-DOF single-slave teleoperator is found as

$$
\left[\begin{array}{c}
F_{h} \\
F_{e}
\end{array}\right]=\left[\begin{array}{cc}
\mathbf{C}_{m}+\mathbf{Z}_{m} & -\mathbf{C}_{4} \\
-\mathbf{C}_{1} & \mathbf{C}_{s}+\mathbf{Z}_{s}
\end{array}\right]\left[\begin{array}{l}
V_{h} \\
V_{e}
\end{array}\right]
$$

Now, let us investigate the stability of the teleoperator via Theorem 9. With $s=j \omega$, the symmetrization conditions of $\mathrm{A}$ and $\mathrm{B}$ boil down to the following conditions involving the control gains and the frequency $\omega$ :

$$
\begin{aligned}
& k_{v 1 x y}=k_{p 1 x y}=k_{v 1 x z}=k_{p 1 x z}=0 \\
& \omega^{2}\left(k_{v 1 y z} k_{v 4 y y}-k_{v 4 y z} k_{v 1 y y}\right)+j \omega\left(k_{v 4 y z} k_{p 1 y y}\right. \\
& \left.+k_{p 4 y z} k_{v 1 y y}-k_{v 1 y z} k_{p 4 y y}-k_{p 1 y z} k_{v 4 y y}\right) \\
& +k_{p 4 y z} k_{p 1 y y}-k_{p 1 y z} k_{p 4 y y}=0 \\
& \omega^{2}\left(k_{v 1 z z} k_{v 4 y z}-k_{v 4 z z} k_{v 1 y z}\right)+j \omega\left(k_{v 4 z z} k_{p 1 y z}\right. \\
& \left.+k_{p 4 z z} k_{v 1 y z}-k_{v 1 z z} k_{p 4 y z}-k_{p 1 z z} k_{v 4 y z}\right) \\
& +k_{p 4 z z} k_{p 1 y z}-k_{p 1 z z} k_{p 4 y z}=0
\end{aligned}
$$

Conditions (18)-(20) will be fulfilled for all frequencies $\omega$ if the gains of the PD controllers (16) satisfy

$$
\begin{aligned}
& k_{v 1 x y}=k_{p 1 x y}=k_{v 1 x z}=k_{p 1 x z}=0 \\
& \frac{k_{v 4 y y}}{k_{v 1 y y}}=\frac{k_{v 4 y z}}{k_{v 1 y z}}=\frac{k_{v 4 z z}}{k_{v 1 z z}}=\frac{k_{p 4 y y}}{k_{p 1 y y}}=\frac{k_{p 4 y z}}{k_{p 1 y z}}=\frac{k_{p 4 z z}}{k_{p 1 z z}}
\end{aligned}
$$

It is easy to see that, under (21), all the elements of the impedance matrix (17) have only a simple pole on the imaginary axis, thus satisfying Condition C. Analysis of the residues according to Condition D leads to the following additional constraints:

$$
\begin{aligned}
& k_{p m x x} \geq 0, \quad k_{p m y y} \geq 0, \quad k_{p m z z} \geq 0 \\
& k_{p s x x} \geq 0, \quad k_{p s y y} \geq 0, \quad k_{p s z z} \geq 0 \\
& k_{p m y y} k_{p m z z}-k_{p m y z}^{2} \geq 0 \\
& \left(k_{p 1 x x} k_{p 4 x x}-k_{p m x x} k_{p s x x}\right) \geq 0 \\
& k_{p 1 y z} k_{p m y y}=k_{p m y z} k_{p 1 y y}, \quad k_{p s x y}=0 \\
& k_{p 1 x x}=k_{p s x x}, \quad k_{p 1 y y}=k_{p s y y}, \quad k_{v 1 x x}=k_{v s x x} \\
& k_{v 1 y y}=k_{v s y y}, \quad k_{p 4 x x}=k_{p m x x}, \quad k_{v 4 x x}=k_{v m x x}
\end{aligned}
$$

Now, let us deal with Condition E of Theorem 9. Condition (9a) turns out to state

$$
\begin{aligned}
& k_{v m x x} \geq 0, \quad k_{v m y y} \geq 0, \quad k_{v m z z} \geq 0 \\
& k_{v s x x} \geq 0, \quad k_{v s y y} \geq 0, \quad k_{v s z z} \geq 0
\end{aligned}
$$

Under (21) and (22), the second leading principal minor condition, i.e., $(9 \mathrm{~b})$, gives

$$
k_{v m x x} k_{v m y y} \geq 0
$$

Similarly, the third leading principal minor condition requires

$$
k_{v m x x}\left(k_{v m y y} k_{v m z z}-k_{v m y z}^{2}\right) \geq 0
$$

The fourth leading principal minor condition mandates

$$
-\left(k_{p m x x} k_{v s x x}-k_{v m x x} k_{p s x x}\right)^{2} \geq 0
$$

Condition (26) will be fulfilled if the PD control gain satisfy

$$
\frac{k_{p m x x}}{k_{v m x x}}=\frac{k_{p s x x}}{k_{v s x x}}
$$

The fifth leading principal minor condition mandates

$$
k_{v s x y}=0
$$

Finally, under above stability conditions, the fifth leading principal minor condition and the sixth leading principal minor condition, i.e., (9c) always positive.

All in all, a sufficient, frequency-independent, and compact condition set for stability of the above-described teleoperator is

$k_{v 1 x y}=k_{p 1 x y}=k_{v 1 x z}=k_{p 1 x z}=k_{p s x y}=k_{v s x y}=0$

$\frac{k_{v 4 y y}}{k_{v 1 y y}}=\frac{k_{v 4 y z}}{k_{v 1 y z}}=\frac{k_{v 4 z z}}{k_{v 1 z z}}=\frac{k_{p 4 y y}}{k_{p 1 y y}}=\frac{k_{p 4 y z}}{k_{p 1 y z}}=\frac{k_{p 4 z z}}{k_{p 1 z z}}$

$k_{p m y y} k_{p m z z}-k_{\text {pmyz }}^{2} \geq 0, \quad k_{v m y y} k_{v m z z}-k_{v m y z}^{2} \geq 0$

$k_{p 1 x x}=k_{p s x x}, \quad k_{p 1 y y}=k_{p s y y}, \quad k_{v 1 x x}=k_{v s x x}$

$k_{v 1 y y}=k_{v s y y}, \quad k_{p 4 x x}=k_{p m x x}, \quad k_{v 4 x x}=k_{v m x x}$

$\frac{k_{p m x x}}{k_{v m x x}}=\frac{k_{p s x x}}{k_{v s x x}}, \quad k_{p 1 y z} k_{p m y y}=k_{p m y z} k_{p 1 y y}$

where all control gains are nonnegative. The ratios in (29) are merely artifacts of our presentation of the stability conditions meaning that division by zero can be avoided.

\subsection{Simulations}

In general, for checking the stability of a two-port network, the port \#2 (environment port) can be connected to passive terminations while the input energy at the port \#1 (operator port) is measured. The teleoperator is absolutely stable if and only if, at all times $t>0$, we have:

$$
E_{s}(t)=\int_{0}^{t} F_{1}^{T}(\tau) V_{1}(\tau) \mathrm{d} \tau \geq 0 .
$$

The position-position (1+2)-DOF dual-master/3-DOF single-slave teleoperation system is simulated in MATLAB/ Simulink. There is no time delay in the communication channel between the masters and the slave. The 1-DOF, 2-DOF, and 3-DOF robots as the masters and the slave are modeled as in (14) with $M_{m x x}=1.7, M_{m y y}=1.9$, $M_{m z z}=1.3, M_{m y z}=0.3, M_{s x x}=23, M_{s x y}=5$, $M_{s y y}=5.6, M_{s z z}=15 M_{s x z}=0.5$ and, $M_{s y z}=1.3$. According to (29), the stability of the position-position teleoperation system should depend on the controllers 
Table 1. The controllers gains of the 1-DOF + 2-DOF dual-master/3-DOF signal-slave teleoperation system used in simulations.

\begin{tabular}{l|llllll}
\hline \hline \multirow{5}{*}{ Master } & $k_{p m x x}$ & 400 & $k_{\text {pmyy }}$ & 300 & $k_{p m z z}$ & 250 \\
& $k_{v m x x}$ & 80 & $k_{v m y y}$ & 60 & $k_{v m z z}$ & 50 \\
& $k_{p 4 x x}$ & 400 & $k_{p 4 y y}$ & 300 & $k_{p 4 y z}$ & 30 \\
& $k_{v 4 x x}$ & 80 & $k_{v 4 y y}$ & 60 & $k_{v 4 y z}$ & 10 \\
& $k_{p 4 z z}$ & 250 & $k_{p m y z}$ & 30 & $(300)$ & \\
& $k_{v 4 z z}$ & 50 & $k_{v m y z}$ & 10 & & \\
\hline \multirow{5}{*}{ Slave } & $k_{p s x x}$ & 200 & $k_{p s y y}$ & 150 & $k_{p s z z}$ & 125 \\
& $k_{v s x x}$ & 40 & $k_{v s y y}$ & 30 & $k_{v s z z}$ & 25 \\
& $k_{p s x y}$ & 0 & $k_{p s x z}$ & 15 & $k_{p s y z}$ & 15 \\
& $k_{v s x y}$ & 0 & $k_{v s x z}$ & 20 & $k_{v s y z}$ & 5 \\
& $k_{p 1 x x}$ & 200 & $k_{p 1 y y}$ & 150 & $k_{p 1 z z}$ & 125 \\
& $k_{v 1 x x}$ & 40 & $k_{v 1 y y}$ & 30 & $k_{v 1 z z}$ & 25 \\
& $k_{p 1 x y}$ & 0 & $k_{p 1 x z}$ & 0 & $k_{p 1 y z}$ & 15 \\
& $k_{v 1 x y}$ & 0 & $k_{v 1 x z}$ & 0 & $k_{v 1 y z}$ & 5 \\
\hline
\end{tabular}

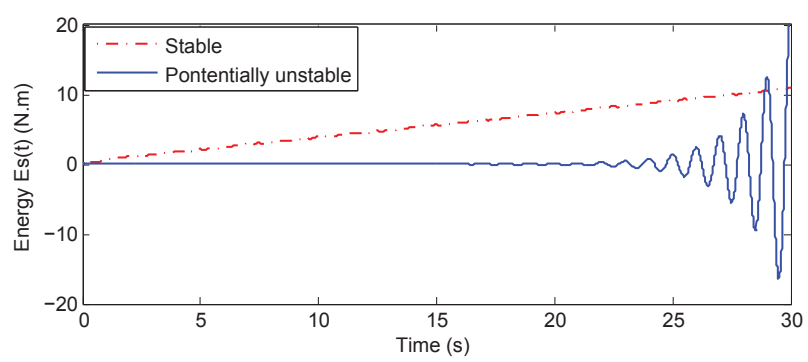

Fig. 4. Simulation results for the $(1+2)-$ DOF dualmaster/3-DOF single-slave teleoperation system. Input energy at port 1 (the masters' port) is shown while port 2 (the slave's port) is connected to an LTI passive termination. The control gains are listed in Table 1 for the stable case with $K_{p m y z}=30$ and for the potentially unstable case with $K_{p m y z}=300$.

gains. In the simulations, the controllers gains were chosen according to Table 1.

To check the stability of the network, the slave ports is connected to the LTI terminations

$$
\mathbf{T}_{2}=\left[\begin{array}{ccc}
\frac{9}{s+1} & -\frac{2}{s+2} & -\frac{1}{s+3} \\
-\frac{2}{s+2} & \frac{5}{s+1} & \frac{2}{s+1} \\
-\frac{1}{s+3} & \frac{2}{s+1} & \frac{4}{s+1}
\end{array}\right]
$$

which is passive. Port 1 is open and we apply sine-wave inputs $f_{h x}$ to the master 1 and $f_{h y}$ and $f_{h z}$ to the master 2. The input energy $E_{s}(t)$ in (30) is plotted in Figure 4. As it can be seen, if the control gains are selected according to (29), e.g., as listed in Table 1 , with $K_{\text {pmyz }}=30$, then the input energy at port 1 is non-negative at all times, indicating the stability of the teleoperator. However, when we change $K_{p m y z}$ to 300 , which violates (29), the input energy $E_{s}(t)$ will become negative at least for a period of time, indicating potential instability of the teleoperator. The above show that there is agreement between the theoretical stability condition (29) and the simulations.

\section{CONCLUSIONS}

This paper presented a closed-form and easy-to-use stability criterion for a $(1+2)$-DOF dual-master/3-DOF singleslave teleoperation systems. Through a case study, we elaborated on its application in stability analysis of teleoperation systems involving several master haptic interfaces with complementary degrees of freedom. Through simulations, the proposed stability criterion was validated.

\section{ACKNOWLEDGMENT}

This research was supported by the Natural Sciences and Engineering Research Council (NSERC) of Canada, by the Natural Science Foundation of China (NFSC, Grant No. 51277022), and by the China Scholarship Council (CSC) under grant [2011]3005.

\section{REFERENCES}

Hager, G.D. (2010). Human-machine cooperative manipulation with vision-based motion constraints. Springer London, 401, 55-70.

Khalil, H.K. (2002). Nonlinear Systems. Prentice Hall.

Kim, K., cavusoglu, M., and Chung, W.K. (2007). Quantitative comparison of bilateral teleoperation systems using mu-synthesis. IEEE Transactions on Robotics, 23(4), 776-789.

Li, J., Tavakoli, M., and Huang, Q. (2013a). Stability analysis of trilateral haptic collaboration. IEEE World Haptics Conference 2013, $611-616$.

Li, J., Tavakoli, M., Mendez, V., and Huang, Q. (2013b). Conservatism of passivity criteria for stability analysis of trilateral haptic systems. IEEE World Haptics Conference 2013, $633-638$.

Tanaka, H., Ohnishi, K., Nishi, H., Kawai, T., Morikawa, Y., Ozawa, S., and Furukawa, T. (2009). Implementation of bilateral control system based on acceleration control using fpga for multi-dof haptic endoscopic surgery robot. IEEE Transactions on Industrial Electronics, 56(3), 618-627.

Tavakoli, M., Aziminejad, A., Patel, R., and Moallem, M. (2007). High-fidelity bilateral teleoperation systems and the effect of multimodal haptics. IEEE Transactions on Systems, Man, and Cybernetics, Part B: Cybernetics, $37(6), 1512-1528$.

Ueki, S., Kawasaki, H., Ito, S., Nishimoto, Y., Abe, M., Aoki, T., Ishigure, Y., Ojika, T., and Mouri, T. (2012). Development of a hand-assist robot with multi-degreesof-freedom for rehabilitation therapy. IEEE/ASME Transactions on Mechatronics, 17(1), 136-146.

Walker, K., Pan, Y.J., and Gu, J. (2009). Bilateral teleoperation over networks based on stochastic switching approach. IEEE/ASME Transactions on Mechatronics, 14(5), 539-554.

Yamashita, T., Godler, I., Takahashi, Y., Wada, K., and Katoh, R. (1991). Peg-and-hole task by robot with force sensor: Simulation and experiment. In IECON '91, volume 2, 980-985.

Youla, D. (1959). A stability characterization of the reciprocal linear passive n-port. Proc. IRE, 47, 11501151.

Youla, D. (1960). A note on the stability of linear, nonreciprocal n-port. Proc. IRE, 48, 121-122.

Zhai, S. and Milgram, P. (1998). Quantifying coordination in multiple dof movement and its application to evaluating 6 dof input devices. Proceedings of the SIGCHI Conference on Human Factors in Computing Systems, CHI 98, 320-327. 